\title{
A note on quasi-robust cycle bases*
}

\author{
Philipp-Jens Ostermeier \\ Bioinformatics Group, Department of Computer Science, University of Leipzig \\ Härtelstrasse 16-18, D-04107 Leipzig, Germany \\ Marc Hellmuth \\ Max Planck Institute for Mathematics in the Sciences \\ Inselstraße 22, D-04103 Leipzig, Germany \\ Bioinformatics Group, Department of Computer Science, University of Leipzig \\ Härtelstrasse 16-18, D-04107 Leipzig, Germany

\section{Konstantin Klemm} \\ Bioinformatics Group, Department of Computer Science, University of Leipzig \\ Härtelstrasse 16-18, D-04107 Leipzig, Germany

\section{Josef Leydold}

Department of Statistics and Mathematics, Vienna University of Economics and Business Augasse 2-6, A-1090 Wien, Austria

\section{Peter F. Stadler}

Bioinformatics Group, Department of Computer Science and Interdisciplinary Center of Bioinformatics, University of Leipzig

Härtelstrasse 16-18, D-04107 Leipzig, Germany

Max Planck Institute for Mathematics in the Sciences Inselstraße 22, D-04103 Leipzig, Germany

Fraunhofer Institut f. Zelltherapie und Immunologie Perlickstraße 1, D-04103 Leipzig, Germany

Inst. f. Theoretical Chemistry, University of Vienna Währingerstraße 17, A-1090 Wien, Austria

Santa Fe Institute

1399 Hyde Park Rd., Santa Fe, NM 87501, USA

Received 31 March 2009, accepted 5 November 2009, published online 30 November 2009

* The work reported here was initiated at the Workshop on Cycle and Cut Bases in Tübingen, May 16-18, 2008, organized by Katharina Zweig. 


\begin{abstract}
We investigate here some aspects of cycle bases of undirected graphs that allow the iterative construction of all elementary cycles. We introduce the concept of quasi-robust bases as a generalization of the notion of robust bases and demonstrate that a certain class of bases of the complete bipartite graphs $K_{m, n}$ with $m, n \geq 5$ is quasi-robust but not robust. We furthermore disprove a conjecture for cycle bases of Cartesian product graphs.

Keywords: Cycle space, cycle basis, robust, quasi-robust, Kainen's basis, elementary cycle, complete bipartite, Cartesian product.
\end{abstract}

Math. Subj. Class.: 05C10

\title{
1 Introduction
}

Cycle bases [2,9] are not only an interesting characterization of the structure of graphs by themselves but also provide a basis for computational assessments of the cycle structure of a graph. "Cycle-space algorithms", for instance, attempt to construct the set of all elementary cycles of a graph from a cycle basis $\mathcal{B}$ by iteratively computing the symmetric difference of an elementary cycle and a basis cycle, subsequently retaining the result if and only if it is again an elementary cycle. If a cycle basis is robust, this approach is successful [1, 4, 8, 11].

Unfortunately, however, very little is known about robust cycle bases beyond a few very special graph classes: As shown in [4], the boundaries of the faces of an embedded planar graph form a robust cycle basis. Corresponding cycle-space algorithms are given in $[14,4]$. Furthermore, complete graphs have robust bases that are easy to construct explicitly [8]. Robust bases for a small class of cubic graphs are constructed in [11].

There is, at present, neither an efficient algorithm to construct a robust cycle basis for a given input graph, nor is it known whether robust bases always exist. A major obstacle for the investigation of robust cycle bases is the apparent lack of relationships with other classes of cycle bases that have been explored in much more detail in the past [6, 12]. For instance, Dixon and Goodman [3] conjectured that every strictly fundamental cycle basis is robust. A counterexample, however, was given in [13]. A more systematic search for connections [11] showed that robust and fundamental cycle bases are also unrelated.

In this contribution we first disprove two conjectures on robust cycle bases. We then introduce a relaxed notion of robustness that is still sufficient for the construction of efficient cycle space algorithms and show that "quasi-robust" cycle bases can be constructed for complete bipartite graphs.

\section{Preliminaries}

Throughout this contribution, let $G=(V, E)$ be a finite undirected simple 2-connected graph. A path $P$ is a connected graph such that each vertex of $P$ has degree at most 2 . The number of edges in $P$ defines its length. A (generalized) cycle in $G$ is an Eulerian subgraph of $G$, i.e., a subgraph of $G$ in which the degree of every vertex is even. A connected

E-mail addresses: philipp@bioinf.uni-leipzig.de (Philipp-Jens Ostermeier), marc@ bioinf.uni-leipzig.de (Marc Hellmuth), klemm@bioinf.uni-leipzig.de (Konstantin Klemm), josef.leydold@wu.ac.at (Josef Leydold), studla@bioinf.uni-leipzig.de (Peter F. Stadler) 
Eulerian subgraph in which every vertex has degree 2 will be called an elementary cycle. For simplicity, we identify a subset $E^{\prime} \subseteq E$ of edges of $G$ with the subgraph $G\left(E^{\prime}\right):=$ $\left(\bigcup_{e \in E^{\prime}} e, E^{\prime}\right)$ of $G$ that it defines. In particular, we identify cycles with their edge sets. The symmetric difference of two edge sets $E^{\prime}$ and $E^{\prime \prime}$ will be denoted by $E^{\prime} \oplus E^{\prime \prime}$, i.e., we put $E^{\prime} \oplus E^{\prime \prime}:=\left(E^{\prime} \cup E^{\prime \prime}\right) \backslash\left(E^{\prime} \cap E^{\prime \prime}\right)$. It will sometimes be convenient to identify a cycle by the sequence of vertices traversed in one of the two orientations. We write

$$
C=\left\{x_{1} x_{2}, x_{2} x_{3}, \ldots x_{k-1} x_{k}, x_{k} x_{1}\right\}=:\left[x_{1}, x_{2} \ldots, x_{k}\right],
$$

and use the shorthand $x y=\{x, y\}$ to denote edges as pairs of adjacent vertices.

The power set $\mathfrak{P}(E)$ can be regarded as a vector space over $\mathrm{GF}(2)=\{0,1\}$ with vector addition $\oplus$ and the trivial multiplication operator $1 \cdot D=D, 0 \cdot D=\varnothing$. The cycle space $\mathfrak{C}(G)$ is the subspace of $(\mathfrak{P}(E), \oplus, \cdot)$ that consists of the cycles of $G$ (including the "empty cycle" $\varnothing)$, see e.g. [2]. As every 2-connected graph $G$ is connected, the dimension $\operatorname{dim}_{\mathrm{GF}(2)} \mathfrak{C}(G)$ of its cycle space coincides with its cyclomatic number $\mu(G):=|E|-$ $|V|+1$, see e.g. [5].

We call a basis $\mathcal{B}$ of $\mathfrak{C}(G)$ that consists only of elementary cycles a cycle basis of $G$. For every cycle $C$, there is a unique subset $\mathcal{B}_{C} \subseteq \mathcal{B}$ of (elementary) cycles in $\mathcal{B}$ such that $C=\bigoplus_{C^{\prime} \in \mathcal{B}_{C}} C^{\prime}$ holds. For a planar embedding $G^{p}$ of a planar graph $G$, the facial basis is the set of boundaries (elemantary cycles) of $G^{p}$ for all finite open and connected faces.

\section{Robust and quasi-robust bases}

In the following we build on the discussion in [11], which is based on [8] but uses a somewhat different terminology.

Definition 3.1. A sequence $\mathcal{S}=\left(C_{1}, C_{2}, \ldots, C_{k}\right)$ of (not necessarily pairwise distinct) elementary cycles is well-arranged if, for each $j \leq k$, the partial sum $Q_{j}=\bigoplus_{i=1}^{j} C_{i}$ is an elementary cycle.

The sequence $\mathcal{S}$ is strictly well-arranged if, for $2 \leq j \leq k, C_{j} \cap Q_{j-1}$ is a path consisting of at least one edge.

By construction, strictly well-arranged cycle sequences are well-arranged, while the converse is not true $[8,11]$. A small example of a sequence $\mathcal{S}$ which is well-arranged but not strictly is shown in Figure 1.

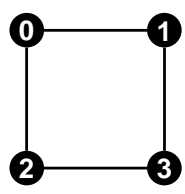

$C_{1}$

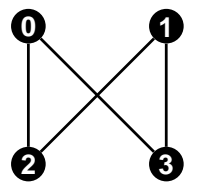

$\mathrm{C}_{2}$

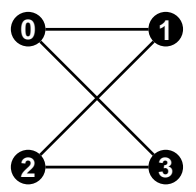

$C_{1} \oplus C_{2}$

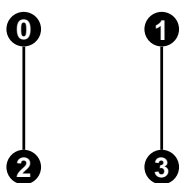

$C_{2} \cap Q_{1}$

Figure 1: Sequence $\mathcal{S}=\left(C_{1}, C_{2}\right)$ which is well-arranged, but not strictly well-arranged.

Definition 3.2. A cycle basis $\mathcal{B}$ is (strictly) quasi-robust if, for each elementary cycle $C \in$ $\mathfrak{C}(G)$ there is a (strictly) well-arranged sequence of cycles $\mathcal{S}_{C}=\left(C_{1}, C_{2}, \ldots, C_{k_{C}}, C\right)$ 
with $C_{i} \in \mathcal{B}, 1 \leq i \leq k_{C}$, and $Q_{k_{C}}=\bigoplus_{i=1}^{k_{C}} C_{i}=C$. The basis $\mathcal{B}$ is (strictly) robust if for each elementary cycle $C$, the (strictly) well-arranged sequence $\mathcal{S}_{C}$ can be chosen such that all cycles in $\mathcal{S}_{C} \backslash\{C\}$ are linearly independent. In particular, it holds that $C_{i} \neq C_{j}$ if $i \neq j$ for all $C_{i}, C_{j} \in S_{C}$.

A cycle basis $\mathcal{B}$ of a graph $G$ can be associated with an undirected graph $\Gamma_{\mathcal{B}}$ whose vertices are all elementary cycles in $G$ (including the empty elementary cycle $\varnothing$ ). An edge in $\Gamma_{\mathcal{B}}$ connects two elementary cycles $C^{\prime}$ and $C^{\prime \prime}$ if and only if $C^{\prime} \oplus C^{\prime \prime} \in \mathcal{B}$. The next lemma provides a characterization of robust and quasi-robust cycle bases in terms of this graph $\Gamma_{\mathcal{B}}$.

Lemma 3.3. Let $\mathcal{B}$ be a cycle basis of $G$. Then

1. $\mathcal{B}$ is quasi-robust if and only if $\Gamma_{\mathcal{B}}$ is connected.

2. $\mathcal{B}$ is robust if and only if for every elementary cycle $C$, the length of a shortest path in $\Gamma_{\mathcal{B}}$ connecting $C$ with $\varnothing$ equals $\left|\mathcal{B}_{C}\right|$.

Proof. (1) If $\mathcal{B}$ is quasi-robust it follows directly from the definition of $\Gamma_{\mathcal{B}}$ that $\Gamma_{\mathcal{B}}$ is connected. Assume now $\Gamma_{\mathcal{B}}$ is connected. In the following we construct a well-arranged sequence $S_{C}$ according to the definition of quasi-robustness. Since $\Gamma_{\mathcal{B}}$ is connected there is for every elementary cycle $C$ a path $P=\left(C^{\prime}, C_{1}, \ldots, C_{k}, C\right)$ in $\Gamma_{\mathcal{B}}$ to an element $C^{\prime}$ with $C^{\prime} \in \mathcal{B}$. Moreover since $\left\{C^{\prime}, C_{1}\right\} \in E\left(\Gamma_{\mathcal{B}}\right)$ we can conclude that $Z_{1}=C^{\prime} \oplus C_{1} \in \mathcal{B}$. Notice that $C_{1}=C^{\prime} \oplus Z_{1}$. Again since $\left\{C_{1}, C_{2}\right\} \in E\left(\Gamma_{\mathcal{B}}\right)$ it follows that $Z_{2}=C_{1} \oplus C_{2} \in \mathcal{B}$ and thus $C_{2}=C^{\prime} \oplus Z_{1} \oplus Z_{2}$. By induction we end with $C=C^{\prime} \oplus \bigoplus_{i=1}^{k+1} Z_{i}$ which provides us a well-arranged sequence $S_{C}=\left(C^{\prime}, Z_{1}, \ldots, Z_{k}, Z_{k+1}, C\right)$.

(2) The path length cannot be smaller than the number $\left|\mathcal{B}_{C}\right|$ of basis cycles necessary to represent $C$. If $C$ can be reached via no more than $\left|\mathcal{B}_{C}\right|$ intermediate cycles, these must be reached via a well-arranged ordering of $\mathcal{B}_{C}$ because each basis cycle in $\mathcal{B}_{C}$ must be used at least once.

\section{Counterexamples}

\subsection{Kainen's basis of $K_{m, n}$}

Let us denote by $V_{1} \dot{\cup} V_{2}$ the vertex bipartition of the complete bipartite graph $K_{m, n}$. We fix two vertices $p \in V_{1}$ and $q \in V_{2}$ and consider the set of quadrangles

$$
\mathcal{B}^{p, q}=\left\{\{p q, p y, q x, x y\} \mid x \in V_{1} \backslash\{p\}, y \in V_{2} \backslash\{q\}\right\} .
$$

Since every edge $x y$ appears only in one single basis quadrangle, we see immediately that $\mathcal{B}^{p, q}$ is linearly independent. Furthermore, $\left|\mathcal{B}^{p, q}\right|=\left(\left|V_{1}\right|-1\right) \times\left(\left|V_{2}\right|-1\right)=\mu\left(K_{m, n}\right)$, i.e., $\mathcal{B}^{p, q}$ is basis of $\mathfrak{C}\left(K_{m, n}\right)$, to which we will refer as Kainen's basis.

In [8], it was argued that $\mathcal{B}^{p, q}$ is robust. Here we give a counterexample. In $K_{5,5}$, consider the cycle $C$ as shown in Figure 2. As indicated in the caption of Figure 2, its generating set $\mathcal{B}_{C}^{1,8}$ w.r.t. the Kainen basis $\mathcal{B}^{1,8}$ cannot be well-arranged, hence $\mathcal{B}^{1,8}$ is not robust. We can make an even stronger statement: None of the Kainen bases of $K_{5,5}$ are robust, which follows directly from the observation that there is is an automorphism $\pi$ of $K_{5,5}$ with $\pi(p)=1$ and $\pi(q)=8$ with $p \in V_{1}$ and $q \in V_{2}$. The pre-image of an elemantary cycle $C, \pi^{-1}(C)$, is necessarily again an elementary cycle. The relationship of 


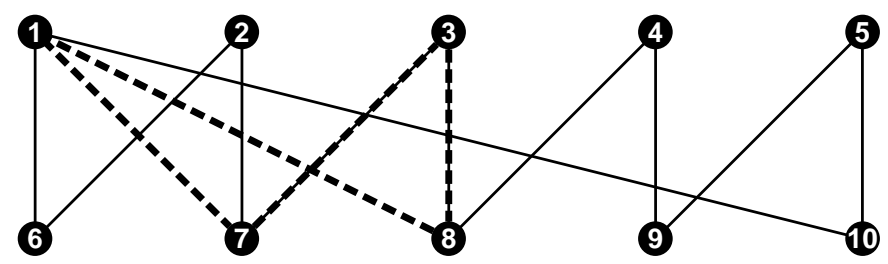

Figure 2: Counterexample for Kainen's assertion. Consider the basis $\mathcal{B}^{1,8}$ of $K_{5,5}$ and consider the cycle $C=[1,6,2,7,3,8,4,9,5,10]$ outlined by the full line. The generating set of $C$ is $\mathcal{B}_{C}^{1,8}=\left\{C_{4,9}, C_{3,7}, C_{5,10}, C_{2,6}, C_{2,7}, C_{5,9}\right\}$, where $C_{x y}=[1,8, x, y]$. As an example one elementary cycle $C_{3,7}$ is shown as dashed lined subgraph. Assume now an arbitrary sequence $\left(C_{1}, \ldots, C_{6}\right)$ with $C_{i} \in \mathcal{B}_{C}^{1,8}$. One easily checks that $C \oplus C_{j}=$ $\left(\bigoplus_{i=1}^{6} C_{i}\right) \oplus C_{j}=\bigoplus_{i=1, i \neq j}^{6} C_{i}$ is not an elementary cycle for each $C_{j} \in \mathcal{B}_{C}^{1,8}$ and for all such sequences. Therefore, there is no well-arranged ordering of $\mathcal{B}_{C}^{1,8}$, and consequently $\mathcal{B}^{1,8}$ is not robust.

$\pi^{-1}(C)$ and $\mathcal{B}^{p, q}$ is the same as that of $C$ and $\mathcal{B}^{1,8}$, hence the generating set of $\pi^{-1}(C)$ cannot be well-arranged, implying that $\mathcal{B}^{p, q}$ is also not robust.

We can extend this negative result to all complete bipartite graphs $K_{m, n}$ with $m, n \geq 5$. By the same symmetry argument, it is always sufficient to consider the Kainen basis $\mathcal{K}=$ $\mathcal{B}^{0,0^{\prime}}$ with arbitrary fixed $0 \in V_{1}=\{0,1, \ldots, m-1\}$ and $0^{\prime} \in V_{2}=\left\{0^{\prime}, 1^{\prime}, \ldots,(n-1)^{\prime}\right\}$. Furthermore, $K_{5,5}$ is an induced subgraph of $K_{m, n}$ for all $m, n \geq 5$. If 0 and $0^{\prime}$ are vertices of $K_{5,5} \subset K_{m, n}$, then the Kainen basis of $K_{m, n}$ contains that of the induced $K_{5,5}$. The elementary cycle $C$ of Figure 2 is also an elementary cycle in $K_{m, n}$ and its basis decomposition $\mathcal{B}_{C}$ w.r.t. the Kainen basis of $K_{m, n}$ consists of the same basis elementary cycles as on $K_{5,5}$. Hence $\mathcal{B}_{C}$ cannot be well-arranged on $K_{m, n}$ for $n, m \geq 5$.

Contrary to the counterexample above, we will show below that Kainen's assertion is true for small complete bipartite graphs.

Lemma 4.1. The Kainen basis of $K_{m, n}$ with $m \leq 4$ and $n \leq 5$ is strictly robust.

Proof. If $n, m \leq 2$ there is nothing to show since $\mathfrak{C}\left(K_{1,1}\right)=\mathfrak{C}\left(K_{1,2}\right)=\emptyset$ and $K_{2,2}$ consist of a single cycle. Since the longest cycles in $K_{m, n}$ have length $2 \min \{m, n\}$, we only need to consider cycles of length 4,6 , and 8 . In the following, we show that for each type of cycle there is a well-arranged order of their Kainen basis elements. The correctness can be checked by direct computation in each case.

\section{Cycles of length 4 .}

1. $C$ contains $00^{\prime}$. Then $C \in \mathcal{K}$ and there is nothing to show.

2. $C$ contains exactly one of the vertices 0 or $0^{\prime}$. W.l.o.g., $0 \in C$. Then $C=$ $\left[0, i^{\prime}, j, j^{\prime}\right]=C_{j, i^{\prime}} \oplus C_{j, j^{\prime}}$ and hence $\mathcal{B}_{C}=\left\{C_{j, i^{\prime}}, C_{j, j^{\prime}}\right\}$ can be strictly wellarranged.

3. $C$ contains neither 0 nor $0^{\prime}$. All such elementary cycles are of the form $C=\left[i, i^{\prime}, j, j^{\prime}\right]$. Its strictly well-arranged sequence is $\mathcal{S}_{C}=\left(C_{j, i^{\prime}}, C_{j, j^{\prime}}\right.$, $\left.C_{i, j^{\prime}}, C_{i, i^{\prime}}, C\right)$. 


\section{Cycles of length 6.}

1. $C$ contains the edge $00^{\prime}$. Such elementary cycles have the form $C=$ $\left[0,0^{\prime}, i, i^{\prime}, j, j^{\prime}\right]$. Its strictly well-arranged sequence is $S_{C}=\left(C_{j, j^{\prime}}, C_{j, i^{\prime}}\right.$, $\left.C_{i, i^{\prime}}, C\right)$.

2. $C$ includes the "fixed vertices" 0 and $0^{\prime}$ but not the edge $00^{\prime}$. Such elementary cycles have the form $C=\left[0, i^{\prime}, i, 0^{\prime}, j, j^{\prime}\right]$. Its strictly well-arranged sequence is $S_{C}=\left(C_{i, i^{\prime}}, C_{j, j^{\prime}}, C\right)$.

3. $C$ contains exactly one of the vertices 0 or $0^{\prime}$. W.1.o.g., $0 \in C$. Such elementary cycles have the form $C=\left[0, i^{\prime}, i, j^{\prime}, j, k^{\prime}\right]$. Its strictly well-arranged sequence is $S_{C}=\left(C_{j, k^{\prime}}, C_{j, j^{\prime}}, C_{i, j^{\prime}}, C_{i, i^{\prime}}, C\right)$.

4. $C$ contains neither 0 nor $0^{\prime}$. Such elementary cycles have the form $C=$ $\left[i, i^{\prime}, j, j^{\prime}, k, k^{\prime}\right]$. Its strictly well-arranged sequence is $S_{C}=\left(C_{j, i^{\prime}}, C_{j, j^{\prime}}\right.$, $\left.C_{k, j^{\prime}}, C_{k, k^{\prime}}, C_{i, k^{\prime}}, C_{i, i^{\prime}}, C\right)$.

\section{Cycles of length 8 .}

In our treatment these elementary cycles can only occur in $K_{4,4}$ and $K_{4,5}$. The case that $C$ contains neither 0 nor $0^{\prime}$ therefore does not appear.

1. $C$ contains $00^{\prime}$. All such elementary cycles $C$ are of the form $C=$ $\left[0,0^{\prime}, i, i^{\prime}, j, j^{\prime}, k, k^{\prime}\right]$. Its strictly well-arranged sequence is $S_{C}=\left(C_{j, i^{\prime}}, C_{j, j^{\prime}}\right.$, $\left.C_{k, j^{\prime}}, C_{k, k^{\prime}}, C_{i, i^{\prime}}, C\right)$.

2. $C$ includes $0 \in V_{1}$ and $0^{\prime} \in V_{2}$ but not the edge $00^{\prime}$. In this case we have to distinguish two kinds of elementary cycles: $C=\left[0, i^{\prime}, i, 0^{\prime}, j, j^{\prime}, k, k^{\prime}\right]$ or $D=$ $\left[0, i^{\prime}, i, j, j^{\prime}, 0^{\prime}, k, k^{\prime}\right]$. The corresponding strictly well-arranged sequences are $S_{C}=\left(C_{k, k^{\prime}}, C_{k, j^{\prime}}, C_{j, j^{\prime}}, C_{i, i^{\prime}}, C\right)$ and $S_{D}=\left(C_{i, i^{\prime}}, C_{i, j^{\prime}}, C_{j, j^{\prime}}, C_{k, k^{\prime}}, D\right)$.

3. $C$ contains exactly one of the vertices 0 or $0^{\prime}$. W.l.o.g., $0 \in C$. All such elementary cycles are of the form $C=\left[0, i^{\prime}, i, j^{\prime}, j, k^{\prime}, k, l^{\prime}\right]$. Its strictly wellarranged sequence is $S_{C}=\left(C_{k, l^{\prime}}, C_{k, k^{\prime}}, C_{j, k^{\prime}}, C_{j, j^{\prime}}, C_{i, j^{\prime}}, C_{i, i^{\prime}}, C\right)$.

\subsection{The Cartesian product $K_{2} \square G$}

In this subsection we will disprove a conjecture for cycle bases of Cartesian product graphs. The vertex set of the Cartesian product $G \square H$ of two graphs $G$ and $H$ is the set $\{(g, h) \mid$ $g \in V(G), h \in V(H)\}$ that is the Cartesian product of the vertex sets of the factors. By definition two vertices $\left(g_{1}, h_{1}\right),\left(g_{2}, h_{2}\right)$ are adjacent in $G \square H$ if $g_{1} g_{2} \in E(G)$ and $h_{1}=h_{2}$ or if $g_{1}=g_{2}$ and $h_{1} h_{2} \in E(H)$. For more detailed information we refer the interested reader to [7].

In [8] it is conjectured that robust cycle bases can be constructed for Cartesian product graphs of the form $T \square G$, where $T$ is a tree, provided a robust basis $\mathcal{R}$ is already known for $G$. More precisely, the basis $\mathcal{R}^{\prime}$ consisting of the known robust basis $\mathcal{R}$ for a single $G$-fiber together with all quadrangles of the form $e \square f, e \in E(T), f \in E(G)$ has been proposed.

In the following we consider the planar graph $G$ shown in Figure 3.

The Cartesian product $K_{2} \square G$ has 18 vertices and 45 edges, i.e., $\mu\left(K_{2} \square G\right)=28$. The basis $\mathcal{R}^{\prime}$ consists of the faces of the facial basis $\mathcal{R}$ and all quadrangles of the form $K_{2} \square f$, $f \in E(G)$. As mentioned in the introduction, any facial basis of a planar graph is robust [4], and hence also quasi-robust. 


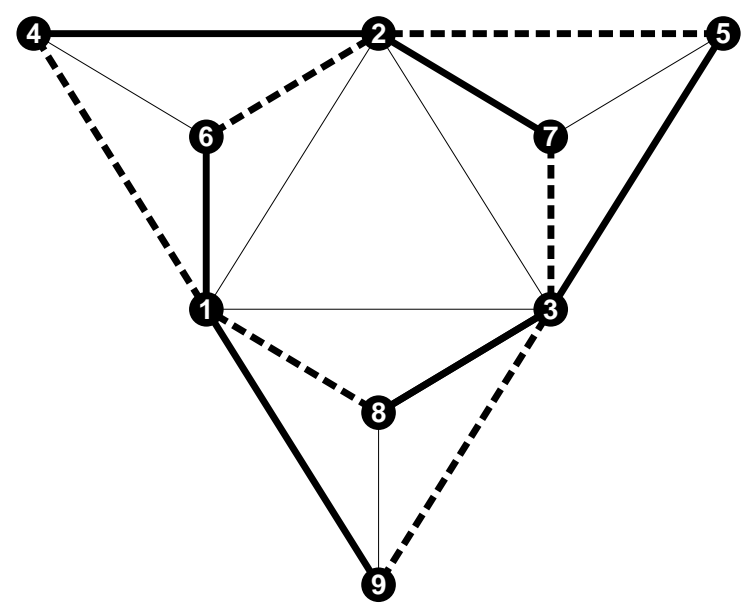

Figure 3: Planar graph $G$. The Cartesian product $K_{2} \square G$ consists of two copies of $G$ in which corresponding vertices are connected by an edge. We consider the elementary cycle $C$ which is partially contained in both the upper copy of $G$ (thick edges) and in the lower copy of $G$ (dashed edges). The elementary cycle $C$ changes from upper to lower edges along a $K_{2}$-fiber wherever a single dashed and a single thick edge meet, i.e., not on vertices 1,2 and 3 .

Now consider the elementary cycle $C$ indicated in Figure 3. For each triangle $D \in \mathcal{R}$, $D \oplus C$ is not an elementary cycle because we always obtain at least one vertex of degree 4. The same is true for any quadrangle $Q=K_{2} \times f$ with $f \in E(G)$. Again we obtain at least one vertex with degree larger than 2 in all cases. Thus the elementary cycle $C$ cannot be constructed from any elementary cycle in $K_{2} \square G$ by adding an element of the basis $\mathcal{R}^{\prime}$. Thus the cycle basis $\mathcal{R}^{\prime}$ is not quasi-robust.

As robust implies quasi-robust, $\mathcal{R}^{\prime}$ is in particular also not a robust basis, disproving Kainen's conjecture.

\section{Quasi-robust basis for $K_{m, n}$}

Theorem 5.1. The Kainen basis $\mathcal{K}$ of $K_{m, n}$ is quasi-robust for all $m, n$.

Proof. We first show that each non-empty elementary cycle $C$ in $K_{m, n}$ that does not include the edge $00^{\prime}$ can be obtained from an elementary cycle that includes the "fixed edge" $00^{\prime}$ by adding a single basis element $D \in \mathcal{K}$.

Case 1: $C$ includes the "fixed vertices" $0 \in V_{1}$ and $0^{\prime} \in V_{2}$ but not the edge $00^{\prime}$.

Claim. There is a $D \in \mathcal{K}$ such that $C \oplus D$ is an elementary cycle containing $00^{\prime}$ satisfying $|C \oplus D|=|C|$.

Proof. W.1.o.g. we can label the vertices so that $C=\left[0, i^{\prime}, i, \ldots, r^{\prime}, r, 0^{\prime}, r+1,(r+\right.$ $\left.1)^{\prime}, \ldots, s, s^{\prime}\right]$. Now we choose $D=\left[0,0^{\prime}, r+1, i^{\prime}\right] \in \mathcal{K}$ and compute $C \oplus D=$ $\left[0,0^{\prime}, r, r^{\prime}, \ldots, j^{\prime}, i, i^{\prime}, r+1,(r+1)^{\prime}, \ldots, s, s^{\prime}, 0\right]$, which we can also interpret as the closed path $\left(0,0^{\prime}, r, r^{\prime}, P\right)$ where $P$ is a path in $K_{p, q}$ which (1) connects 0 and $r^{\prime},(2)$ does not include $0^{\prime}$ and $r$, and (3) has maximum vertex degree 2. Thus $C \oplus D$ 
is an elementary cycle. We have added the two edges $00^{\prime}$ and $i^{\prime}(r+1)$ and removed the edges $0 i^{\prime}$ and $0^{\prime}(r+1)$, hence the length of the cycle $C$ is preserved.

Case 2: $C$ contain exactly one of the vertices 0 or $0^{\prime}$.

Claim. There is a $D \in \mathcal{K}$ such that $C \oplus D$ is an elementary cycle containing the edge $00^{\prime}$ satisfying $|C \oplus D|=|C|$.

Proof. W.l.o.g. we assume that $C$ contains 0 , i.e., $\left[0, i^{\prime}, k, P, j^{\prime}\right]$, where $P$ is a path connecting $k$ and $j^{\prime}$. Set $D=\left[0,0^{\prime}, k, i^{\prime}\right]$. Then $C \oplus D$ is an elementary cycle of length $|C|$ that contains $00^{\prime}$.

Case 3: $C$ contains neither 0 nor $0^{\prime}$.

Claim. There is a $D \in \mathcal{K}$ such that $C \oplus D$ is an elementary cycle of length $|C|+2$ containing $00^{\prime}$.

Proof. Let $i$ and $i^{\prime}$ be any two adjacent vertices of $C$. Then $D=\left[0,0^{\prime}, i, i^{\prime}\right] \in \mathcal{K}$ and $C \oplus D$ is again a elementary cycle. Since we add three edges and delete 1 , its length is $|C|+2$.

Now we show that each elementary cycle of length $|C| \geq 6$ through the edge $00^{\prime}$ can be obtained in two steps from a strictly shorter elementary cycle with the same property.

Claim. Let $C$ be an elementary cycle of length $|C| \geq 6$ including the edge $00^{\prime}$. Then there are basis cycles $D, E \in \mathcal{K}$ such that $C \oplus D$ is an elementary cycle of length $|C \oplus D|=$ $|C|-2$ and $C \oplus D \oplus E$ is an elementary cycle of length $|C \oplus D \oplus E|=|C|-2$ that includes the edge $00^{\prime}$.

Proof. We can write $C$ in the form $C=\left[0,0^{\prime}, j, j^{\prime}, P\right]$, where $P$ is a path of length $|P| \geq 3$ in $K_{m, n}$ that (1) connects 0 and $j^{\prime}$, (2) does not include the edge $0^{\prime} j$, and (3) has maximum vertex degree two. Choosing $D=\left[0,0^{\prime}, j, j^{\prime}\right] \in \mathcal{K}$ we immediately compute $C \oplus D=\left[0, j^{\prime}, P\right]$. Obviously, $D$ is an elementary cycle of length $|C|-2$. Since $P$ has length at least 3 , we can re-write this in the form $C \oplus D=\left[0, j^{\prime}, P\right]=$ $\left[0, j^{\prime}, k, k^{\prime}, Q\right]$, where $Q$ is a path of length $|P|-2 \geq 0$ connecting $k^{\prime}$ and 0 . Now we choose a second basis cycle $E=\left[0,0^{\prime}, k, j^{\prime}\right] \in \mathcal{K}$ and obtain $C \oplus D \oplus E=$ $\left[0,0^{\prime}, k, k^{\prime}, Q\right]$. This is again an elementary cycle, contains the edge $00^{\prime}$ and has length $|C|-2$.

Starting from an arbitrary elementary cycle in $K_{m, n}$, we obtain in at most one step a cycle that runs through $00^{\prime}$. Then we can reduce its length by two in a pair of steps, thereby arriving at a cycle of length 4 through $00^{\prime}$ in not more than $|C|-1$ operations. Since all quadrangles containing $00^{\prime}$ are by construction elements of $\mathcal{K}$, we can reduce every elementary cycle $C$ to $\varnothing$ in no more than $|C|$ steps in such a way that all intermediates are also elementary cycles. Reversing the order, we have thus constructed a well-arranged sub-sequence $\mathcal{S}(C)$ of elements of $\mathcal{K}$ that generates $C$.

Lemma 5.2. The Kainen basis $\mathcal{K}$ of $K_{m, n}$ is not strictly quasi-robust for all $m, n \geq 5$.

Proof. In order to construct a counterexample, we again choose a cycle $C$ of length 10 through 0 and $0^{\prime}$ that does not include the edge $00^{\prime}$. W.l.o.g. we can label the vertices as such that $C=\left[0,1^{\prime}, 3,3^{\prime}, 1,0^{\prime}, 2,4^{\prime}, 4,2^{\prime}\right]$. From Theorem 5.1 it follows that there is a well arranged sequence $\mathcal{S}_{C}$ of the Kainen basis elements. Let $\mathcal{S}_{C}=(\ldots, D, C)$ be any such 
sequence for the particular chosen elementary cycle $C$. First notice that any second-to-last element $D \in \mathcal{S}_{C}$ must include the edges $0 j^{\prime}$ and $0^{\prime} i$, both included in $C$, otherwise $C \oplus D$ would not be an elementary cycle and thus $\mathcal{S}_{C}=(\ldots, D, C)$ would not be well-arranged. Therefore we have exactly four cases: $D_{1,1^{\prime}}, D_{2,2^{\prime}}, D_{1,2^{\prime}}$ and $D_{2,1^{\prime}}$. By symmetry we can reduce these cases to $D_{i, i^{\prime}}$ and $D_{i, j^{\prime}}$.

First notice that $D_{i, i^{\prime}}$ cannot be the second last element of $\mathcal{S}_{C}$, since $C \oplus D_{i, i^{\prime}}$ crumbles down into two elementary cycles.

Thus we only have to consider the case $D_{i, j^{\prime}}$. By direct computing we get the elementary cycle $Q=C \oplus D_{i, j^{\prime}}$ and thus we can observe that $D_{i, j^{\prime}}$ is an element of $\mathcal{S}_{C}$. But on the other hand we can observe that $Q \cap D_{i, j^{\prime}}=\left\{\left\{0,0^{\prime}\right\},\left\{i, j^{\prime}\right\}\right\}$ consisting of two paths. Thus none of the well-arranged sequences $\mathcal{S}_{C}$ can be strictly well-arranged.

\section{Concluding remarks}

A major obstacle in the analysis of robust cycle bases is that no generally applicable "tools" are known, a difficulty that is confounded by the observation that robustness properties are unrelated to other, better-studied, properties of cycle bases such as fundamentality or minimality [11]. By relaxing the definition to quasi-robustness we abandon the requirement that a cycle must be constructed from the basis elements in its linear representation only. This gives us a generic strategy for constructive proofs to establish quasi-robustness: It suffices to show that every elementary cycle can be reduced by $\oplus$-addition of basis elements to another elementary cycle $C^{\prime}$ that is "closer to" $\varnothing$ w.r.t. to some (partial) ordering of elementary cycles (in our example simply by the length of elementary cycles). As an example, we directly prove the following corollary of the robustness result in [4]:

Lemma 6.1. The facial basis of a planar graph $G$ is quasi-robust.

Proof. Consider a planar embedding $G^{p}$ of $G$. Moreover, for any elementary cycle $D, F_{D}$ is the bounded subset of the plane delimited by $D$. Define a partial order on the elementary cycles such that $C \prec C^{\prime}$ if $C \neq C^{\prime}$ and $F_{C}$ lies inside of $F_{C^{\prime}}$, and set $\varnothing \prec C$ for all elementary cycles. Given an elementary cycle $C$, there is a boundary $H$ of a planar face $F_{H}$ such that $C \oplus H \prec C$ is again an elementary cycle. Since there are only finitely many elementary cycles, every elementary cycle can be reduced to $\varnothing$.

In addition to this technical simplification, quasi-robustness is of interest in its own right in the context of sampling algorithms on the cycle space: quasi-robustness is necessary and sufficient for ergodicity of the Markov chains considered in [10].

We have briefly touched upon the "strict" variants of robustness and quasi-robustness. It stands to reason that the additional geometric constraint - intersection of elementary cycles with a basis cycle in a single path - further complicates constructive proofs. Again, strict quasi-robustness seems much easier to handle than strict robustness.

\section{Acknowledgements}

The authors thank the anonymous referees for their valuable comments. This work was partially supported by a grant of the DFG (Proj No. STA850/5-1) and the VolkswagenStiftung. 


\section{References}

[1] F. Berger, C. Flamm, P. M. Gleiss, J. Leydold and P. F. Stadler, Counterexamples in chemical ring perception, J. Chem. Inf. Comput. Sci. 44 (2004), 323-331.

[2] W.-K. Chen, On vector spaces associated with a graph, SIAM J. Appl. Math. 20 (1971), 525529.

[3] E. T. Dixon and S. E. Goodman, An algorithm for the longest cycle problem, Networks 6 (1976), 139-146.

[4] U. Doğrusöz and M. Krishnamoorthy, Enumerating all cycles of a planar graph, J. Parallel Algor. Appl. 10 (1996), 21-36.

[5] L. Euler, Elementa doctrinae solidorum, Novi Comm. Acad. Sci. Imp. Petropol. 4 (1752), 109140.

[6] D. Hartvigsen and R. Mardon, Cycle bases from orderings and coverings, Disc. Math. 94 (1991), 81-94.

[7] W. Imrich and S. Klavžar, Product Graphs, Wiley-Interscience Series in Discrete Mathematics and Optimization, Wiley-Interscience, New York, 2000.

[8] P. C. Kainen, On robust cycle bases, Electronic Notes Discrete Math. 11 (2002), 430-437.

[9] T. Kavitha, C. Liebchen, K. Mehlhorn, D. rios Michail, R. Rizzi, T. Ueckerdt, and K. A. Zweig, Cycle bases in graphs: Characterization, algorithms, complexity, and applications, Computer Science Review 3 (2009), 199-243.

[10] K. Klemm and P. F. Stadler, Statistics of cycles in large networks, Physical Review E (Statistical, Nonlinear, and Soft Matter Physics) 73 (2006), 025101.

[11] K. Klemm and P. F. Stadler, A note on fundamental, non-fundamental, and robust cycle bases, Discrete Appl. Math. 157 (2009), 2432-2438.

[12] C. Liebchen and R. Rizzi, Classes of cycle bases, Discrete Appl. Math. 155 (2007), 337-355.

[13] M. M. Sysło, Characterizations of outerplanar graphs, Discrete Math. 26 (1979), 47-53.

[14] M. M. Sysło, An efficient cycle vector space algorithm for listing all cycles of a planar graph, SIAM J. Comput. 10 (1981), 797-808. 